\title{
Brillouin-Wigner perturbation theory in open electromagnetic systems
}

\author{
E. A. Muljarov ${ }^{1}$, W. Langbein ${ }^{1}$ and R. Zimmermann ${ }^{2}$
}

1 School of Physics and Astronomy, Cardiff University, The Parade, CF243AA, Cardiff, United Kingdom

ㄱ. Institut für Physik der Humboldt-Universität zu Berlin, Newtonstrasse 15, D-12489 Berlin, Germany

\author{
PACS 03.50.De - Classical electromagnetism, Maxwell equations \\ PACS 42.25.-p - Wave optics \\ PACS 03.65. Nk - Scattering theory \\ PACS 41.20.-q-Applied classical electromagnetism
}

\begin{abstract}
A Brillouin-Wigner perturbation theory is developed for open electromagnetic systems which are characterised by discrete resonant states with complex eigenenergies. Since these states are exponentially growing at large distances, a modified normalisation is introduced that allows a simple spectral representation of the Green's function. The perturbed modes are found by solving a linear eigenvalue problem in matrix form. The method is illustrated on exactly solvable one- and three-dimensional examples being, respectively, a dielectric slab and a microsphere.
\end{abstract}

Eigenvalue problems are often not analytically solvable, and approximate solution schemes are employed, which use a known (ideally analytic) solution of a similar problem, differing from the problem of interest by the 'perturbation'. The most well known scheme is the RayleighSchrödinger perturbation theory [1, which writes the solution as series expansion in terms of the perturbation involving sums over all eigenstates, and thus introduces the notion of perturbation orders. However, the convergence properties of such series limit the treatable perturbation strength, and the mathematical complexity limits the expansion typically to a few orders. One way to overcome this limitation is to truncate the infinite complete set of unperturbed eigenstates to a finite number and then use matrix diagonalisation algorithms to treat the perturbation within this basis exactly, i.e. to all orders. In quantum mechanics, this method is called Brillouin-Wigner (BW) perturbation theory [2]. If the unperturbed spectrum contains bound states only (as for the harmonic oscillator), it allows to treat the effect of perturbation to any required accuracy by taking a sufficiently large number of states. However, if a continuum of scattering states is important, the use of the BW theory becomes problematic. In electromagnetic systems, such a continuum is often the dominating part of the spectrum and discrete bound states (evanescent waves) are the exception.

Both Rayleigh-Schrödinger and BW perturbation theories are well established when treating Hermitian prob- lems which describe conservative systems having realvalued eigenenergies and orthogonal and normalised wave functions. In open systems instead, an eigenstate acquires a certain linewidth which characterises its finite lifetime, so that the eigenenergy becomes complex-valued [3]. The corresponding eigenfunctions are not orthogonal and grow exponentially in the outer space so that they cannot be normalised by integrating their square modulus [4] [6]. Both the energies and the wave vectors of these states have negative imaginary parts resulting in a wave function $\propto \exp [-\Gamma(t-r / v)](\Gamma=-\operatorname{Im} \omega, v$ is the phase velocity). The latter expression provides a physical picture of the exponential growth as a wave front propagating away from the system which has been excited at an earlier time. In this way stationary bound states become quasi-stationary [5] in open systems due to leakage to the outside. In the literature these states are also known as decaying states [7, resonant states (RS) 8], leaky modes [9], quasi-guided modes [10, quasi-normal modes [11,12, etc. Moreover, going away from the real-energy axis into the complex energy plane, a continuum of stationary scattering states, which is problematic in perturbation theory, can be effectively eliminated from the spectrum and replaced by a countable number of RS [6]. Formally, this is achieved by imposing boundary conditions of no incoming waves, i.e. no waves travelling towards the system.

The Rayleigh-Schrödinger perturbation theory of RS has been developed for quantum-mechanical [7] and elec- 
tromagnetic systems 12 . For weak perturbations, an approximate BW theory limited to degenerate modes only has been reported [13. Also, an exact method of calculation of RS perturbed by a point scatter has recently been developed 14. However, sharp resonances in threedimensional structures cannot be calculated by known methods in electrodynamics. Thus it is especially important to develop a BW perturbation theory, which could treat such resonant states under a perturbation of arbitrary strength and shape. Such a theory was only partially formulated in electrodynamics more than 40 years ago [6] and later on proposed also in the quantum-mechanical context [15, 16, but has not been completed neither applied to numerically solve relevant problems.

In this Letter we present a BW perturbation theory of open electromagnetic systems and validate it on exactly solvable one-dimensional (1D) and three-dimensional (3D) examples, for both weak and strong perturbations. Although the RS have complex energies and wave functions exponentially increasing outside the system [5], orthogonality and normalisation can be established. Then, the expansion of the perturbed system into the RS of unperturbed system can be reduced to the standard BW form of diagonalisation of the sum of a diagonal matrix of the unperturbed system and a perturbation matrix.

To make the idea clear and the derivation transparent we present our BW theory first on an effective 1D problem taking a homogeneous dielectric slab as unperturbed system and then, after bringing it to a general form, use it for a dielectric microsphere.

We consider a dielectric slab in vacuum with thickness $2 a$ which is described by a real dielectric constant along the spatial coordinate $z$ [see the inset in fig. 1(a)]:

$$
\varepsilon(z)=\left\{\begin{array}{cc}
\varepsilon_{s}, & |z|<a \\
1, & |z| \geqslant a
\end{array}\right.
$$

The permeability is $\mu=1$ everywhere throughout this work. For transversal eigenmodes $\mathbf{E}_{n}=\hat{\mathbf{x}} E_{n} / \sqrt{S}(S$ is the normalisation area in the $x y$-plane) having zero inplane wave number, $k_{x}=k_{y}=0$, and time-dependent part given by $\exp \left(-i c k_{n} t\right)$ with the complex frequency $c k_{n}$, the Maxwell equation is reduced to

$$
\left[\frac{d^{2}}{d z^{2}}+\varepsilon(z) k_{n}^{2}\right] E_{n}(z)=0
$$

with the electric field $E_{n}$ changing smoothly (i.e. having a continuous derivative) across the boundaries at $z= \pm a$. Assuming the boundary conditions (BC) of only outgoing waves results in the eigenfunctions

$$
E_{n}(z)=\left\{\begin{array}{lc}
(-1)^{n} A_{n} e^{-i k_{n} z}, & z<-a \\
B_{n}\left[e^{i \sqrt{\varepsilon_{s}} k_{n} z}+(-1)^{n} e^{-i \sqrt{\varepsilon_{s}} k_{n} z}\right], & |z|<a \\
A_{n} e^{i k_{n} z}, & z>a
\end{array}\right.
$$

and the wave number eigenvalues

$$
k_{n}=\frac{1}{2 a \sqrt{\varepsilon_{s}}}(\pi n-i \ln \alpha), \quad n=0, \pm 1, \pm 2 \ldots,
$$

where $\alpha=\left(\sqrt{\varepsilon_{s}}+1\right) /\left(\sqrt{\varepsilon_{s}}-1\right)$. The eigenfunctions $E_{n}$ are orthogonal and normalised in a well-known way [6]

$$
\int_{-a}^{a} \varepsilon_{s} E_{n} E_{m} d z-\frac{E_{n}(a) E_{m}(a)+E_{n}(-a) E_{m}(-a)}{i\left(k_{n}+k_{m}\right)}=\delta_{n m}
$$

which is determining the amplitudes to

$$
A_{n}=\frac{e^{-i k_{n} a}}{\sqrt{a\left(\varepsilon_{s}-1\right)}}, \quad B_{n}=\frac{(-i)^{n}}{2 \sqrt{a \varepsilon_{s}}} .
$$

Let us also construct the unperturbed Green's function (GF) $G\left(z, z^{\prime} ; k\right)$ which satisfies the equation

$$
\left[\frac{d^{2}}{d z^{2}}+\varepsilon(z) k^{2}\right] G\left(z, z^{\prime} ; k\right)=\delta\left(z-z^{\prime}\right),
$$

and obeys the same BC of no incoming waves. As in the case of a square-barrier potential in quantum mechanics [17, this GF can be calculated analytically. Inside the slab $\left(-a<z, z^{\prime}<a\right)$ it has the following explicit form:

$$
\begin{aligned}
G\left(z, z^{\prime} ; k\right)= & \frac{1}{2 i q} \frac{e^{i q\left(a-z_{>}\right)}+\alpha e^{-i q\left(a-z_{>}\right)}}{e^{i q a}+\alpha e^{-i q a}} \\
& \times \frac{e^{i q\left(a+z_{<}\right)}+\alpha e^{-i q\left(a+z_{<}\right)}}{-e^{i q a}+\alpha e^{-i q a}}
\end{aligned}
$$

where $z_{>}=\max \left(z, z^{\prime}\right), z_{<}=\min \left(z, z^{\prime}\right)$, and $q=k \sqrt{\varepsilon_{s}}$. The analytic continuation of the GF into the complex $k$ plane has simple poles at $k=k_{n}$ and $k=0$. Then, similar to the resolvent expansion of a Hermitian operator in the energy plane 19, it can be written, using the MittagLeffler theorem, as a sum of partial fractions [7, 18:

$$
G\left(z, z^{\prime} ; k\right)=\sum_{n} \frac{E_{n}(z) E_{n}\left(z^{\prime}\right)}{2 k_{n}\left(k-k_{n}\right)}+\frac{1}{2 i k}=\sum_{n} \frac{E_{n}(z) E_{n}\left(z^{\prime}\right)}{2 k\left(k-k_{n}\right)}
$$

where the residua were expressed in terms of the normalised eigenmodes eq. (3) and a sum rule $\sum_{n} E_{n}(z) E_{n}\left(z^{\prime}\right) / k_{n}=i$ following from the highfrequency asymptotics of the GF [20] was used.

Let us now consider an arbitrary real perturbation $\Delta \varepsilon(z)$ of the dielectric constant inside the slab. The wave equation for the perturbed resonant states $\mathcal{E}_{\nu}$ and wave numbers $\varkappa_{\nu}$ is given as in eq. (2) by

$$
\left\{\frac{d^{2}}{d z^{2}}+[\varepsilon(z)+\Delta \varepsilon(z)] \varkappa_{\nu}^{2}\right\} \mathcal{E}_{\nu}(z)=0
$$

with $\mathrm{BC} \mathcal{E}_{\nu}(z) \propto \exp \left(i \varkappa_{\nu}|z|\right)$ at $|z|>a$. The new GF $\mathcal{G}\left(z, z^{\prime} ; k\right)$ is related to the unperturbed one via the Dyson equation

$$
\begin{aligned}
\mathcal{G}\left(z, z^{\prime} ; k\right) & =G\left(z, z^{\prime} ; k\right) \\
- & k^{2} \int_{-a}^{a} G\left(z, z^{\prime \prime} ; k\right) \Delta \varepsilon\left(z^{\prime \prime}\right) \mathcal{G}\left(z^{\prime \prime}, z^{\prime} ; k\right) d z^{\prime \prime} .
\end{aligned}
$$

On the other hand, a spectral representation of the perturbed GF, similar to eq. (9), can be used. It is derived 
in the Appendix for an arbitrary profile of the dielectric function inside the slab. Then, following ref. [7] we equate residua at the poles $k=\varkappa_{\nu}$ in eq. (11). This results in the following relationship between the unperturbed and perturbed modes:

$$
\mathcal{E}_{\nu}(z)=\sum_{n} \frac{E_{n}(z) \int_{-a}^{a} E_{n}\left(z^{\prime}\right) \Delta \varepsilon\left(z^{\prime}\right) \mathcal{E}_{\nu}\left(z^{\prime}\right) d z^{\prime}}{2\left(k_{n} / \varkappa_{\nu}-1\right)}
$$

In the interior region $|z|<a$ which contains the perturbation, the perturbed RS can be expanded into the unperturbed ones, exploiting the completeness of the latter,

$$
\mathcal{E}_{\nu}(z)=\sum_{n} b_{n \nu} E_{n}(z)
$$

Substituting this expansion into eq. (12) and equating coefficients at the same basis functions $E_{n}(z)$ results in the following matrix equation:

$$
2 b_{n \nu}=\frac{1}{k_{n} / \varkappa_{\nu}-1} \sum_{m} V_{n m} b_{m \nu} .
$$

Introducing new coefficients $c_{n \nu}=b_{n \nu} \sqrt{k_{n}}$ to symmetrise the matrix problem, we arrive at

$$
\sum_{m}\left(\frac{\delta_{n m}}{k_{n}}+\frac{V_{n m}}{2 \sqrt{k_{n} k_{m}}}\right) c_{m \nu}=\frac{1}{\varkappa_{\nu}} c_{n \nu} .
$$

Equation (15) is the central result in our BW perturbation theory of resonant states. Similar to the BW theory of bound states in quantum mechanics, eq. (15) represents a linear eigenvalue problem which requires diagonalisation of a matrix depending only on the unperturbed spectrum and the perturbation [21. However, instead of real eigenenergies and a Hermitian perturbation matrix characteristic for conservative systems, we are dealing here with complex-valued bare and perturbed eigenvalues, $1 / k_{n}$ and $1 / \varkappa_{\nu}$, respectively, and a complex symmetric perturbation matrix $V_{n m} /\left(2 \sqrt{k_{n} k_{m}}\right)$, where

$$
V_{n m}=\int \Delta \varepsilon(\mathbf{r}) \mathbf{E}_{n}(\mathbf{r}) \cdot \mathbf{E}_{m}(\mathbf{r}) d \mathbf{r} .
$$

While from the formulation of the method both the unperturbed system and the perturbation $\Delta \varepsilon(\mathbf{r})$ can be located inside any finite area, we found that good convergency is achieved for a perturbation within the area of the unperturbed system.

To illustrate the accuracy of the method, we use as unperturbed system the slab with $\sqrt{\varepsilon_{s}}=1.5$. The unperturbed discrete spectrum is given by eq. (44) and shown in fig. $1(a, b)$ by circles with a dot. We introduce a strong non-symmetric perturbation $\Delta \varepsilon=10$ and 100 in the layer $(a / 2<z<a)$ [see the inset in fig. 1(a)], thus increasing in the latter case the refractive index by an order of magnitude. The exact eigenvalues $\varkappa_{\nu}^{\text {exact }}$ are given in the Appendix by eq. (A.9). They are compared in fig. $1(a, b)$ (squares) with our numerical result (crosses) calculated
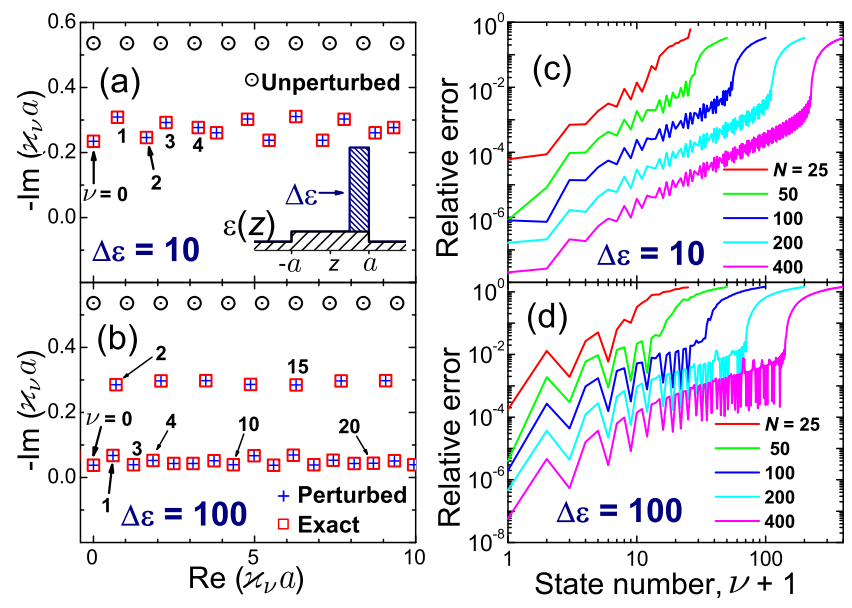

Fig. 1: (colour online) Resonant states of a dielectric slab. Exact and calculated via eq. (15) wave numbers $\varkappa_{\nu}(a, b)$ along with the relative error of the calculation $(c, d)$, for different truncation $N$ of the matrix problem. Unperturbed: RS of a dielectric slab in vacuum (black circles with a dot). The slab with $\varepsilon_{s}=2.25$ occupies the area $-a<z<a$. Perturbed: Exact (red rectangles) and calculated (blue crosses) RS wave numbers $\varkappa_{\nu}$ of the same slab but with a higher refractive index in the layer $(a / 2<z<a)$, the change there being $\Delta \varepsilon=$ $10(a, c)$ or $\Delta \varepsilon=100(b, d)$. Inset: profiles of the dielectric constant of the unperturbed and perturbed systems.

according to eq. (15) where the sums have been truncated at $-N \leq n \leq N$, yielding a matrix dimension of $(2 N+1)$. The relative error $\left|\varkappa_{\nu} / \varkappa_{\nu}^{\text {exact }}-1\right|$ is quantified in fig. $1(c, d)$ for different $N$. States close to the truncation border (i.e. with large values of $|\nu|$ ) are always poorly calculated. However, as the matrix size increases, a state with a given $\nu$ is moving closer to the middle of the matrix, so that its relative error decreases as $N^{-3}$. The exact eigenfunctions (the electric field $\mathcal{E}_{\nu}$ ) are reproduced to a similar accuracy.

Writing the perturbation in the form of eq. (16), the bare eigenmodes $\mathbf{E}_{n}(\mathbf{r})$ are supposed to be normalised as given by eq. (5) in the $1 \mathrm{D}$ case. In quantum-mechanical systems, normalisation of RS was discussed e.g. in refs. 7, 8, 22, A similar approach was used to normalise eigenmodes in spherically symmetric open electromagnetic systems 6, 12,13. Some important details of such normalisation are given in the Appendix along with the spectral representation of the Green's function, see eqs. (A.23) and (A.24). Below we show, however, how this normalisation can be done in the general $3 \mathrm{D}$ case.

The electric field modes $\mathbf{E}_{n}$ obey Maxwell's equation

$$
\nabla \times \nabla \times \mathbf{E}_{n}(\mathbf{r})=k_{n}^{2} \varepsilon(\mathbf{r}) \mathbf{E}_{n}(\mathbf{r})
$$

where the dielectric constant $\varepsilon(\mathbf{r})$ is assumed to deviate from unity only inside a sphere of radius $a$. The perturbation $\Delta \varepsilon(\mathbf{r})$ is located within the same sphere. Multiplying eq. (17) by $\mathbf{E}_{m}(\mathbf{r})$ and integrating the dot product over the sphere of radius $R \geqslant a$ by parts, we obtain the orthogo- 


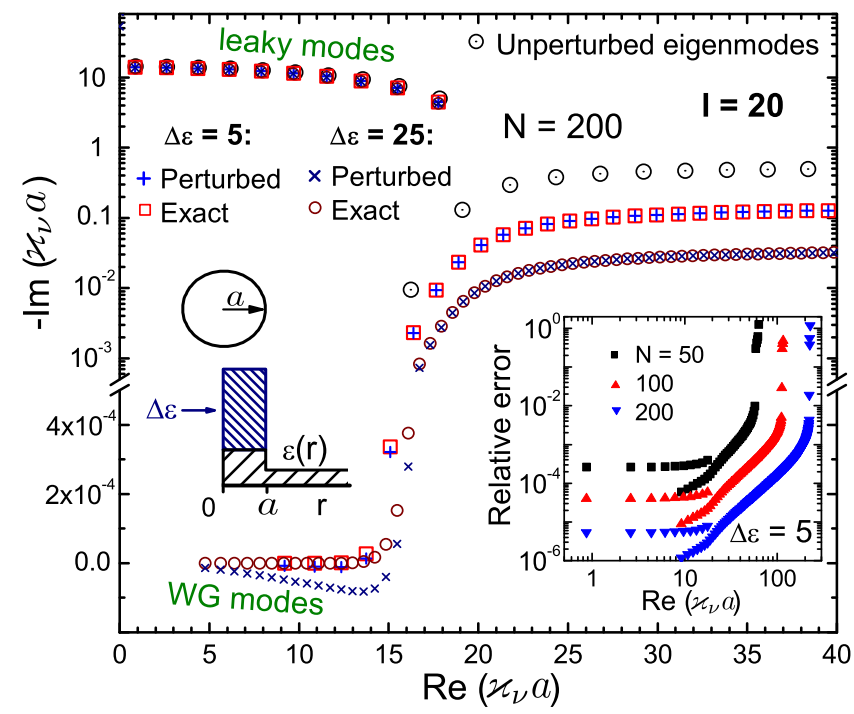

Fig. 2: (colour online) Resonant states of a sphere, with the angular momentum $l=20$ (TE modes). Unperturbed: Exact RS wave numbers $k_{n}$ of a homogeneous dielectric microsphere in vacuum, with radius $a$ and refractive index 1.5 (black circles with a dot). Perturbed: Wave numbers $\varkappa_{\nu}$ of a microsphere with the same radius $a$ but increased dielectric constant $(\Delta \varepsilon=$ 5: red rectangles and blue crosses $+; \Delta \varepsilon=25$ : red circles and blue crosses $\times$ ). Results of eq. (15) are compared with exact values. Left inset: profiles of the dielectric constant of the unperturbed and perturbed systems. Right inset: relative errors of calculation with $\Delta \varepsilon=5$ for different truncation $N$ of the matrix problem.

nality condition

$$
\begin{aligned}
0= & \left(k_{n}^{2}-k_{m}^{2}\right) \int_{R} d \mathbf{r} \varepsilon(\mathbf{r}) \mathbf{E}_{n}(\mathbf{r}) \cdot \mathbf{E}_{m}(\mathbf{r}) \\
& -\int_{R} d S\left(\mathbf{E}_{n} \cdot \frac{\partial \mathbf{E}_{m}}{\partial r}-\mathbf{E}_{m} \cdot \frac{\partial \mathbf{E}_{n}}{\partial r}\right)
\end{aligned}
$$

where the last integral is taken over the surface of the $R$ sphere. Both integrals in eq. (18) are $R$-dependent and divergent at $R \rightarrow \infty$. There is, however, an exact cancellation of these $R$-dependencies thus removing the divergencies [23. In a degenerate case $k_{n}=k_{m}(n \neq m)$, both the volume and the surface integrals vanish by symmetry showing the orthogonality.

Equation (18) is now used as a starting point for the eigenstate normalisation. In the outside range $r>a$, where $\varepsilon(\mathbf{r})=1$, we take advantage of the functional behaviour $\mathbf{E}_{n}(\mathbf{r})=\mathbf{F}_{n}\left(k_{n} \mathbf{r}\right)$ with an auxiliary function $\mathbf{F}_{n}$ being a superposition of vector spherical harmonics [24. The analytic continuation $\mathbf{E}(k, \mathbf{r})$ of the eigenfunction $\mathbf{E}_{n}(\mathbf{r})$ around the point $k_{n}$ in the complex $k$-plane is introduced in such a way that $\mathbf{E}(k, \mathbf{r})=\mathbf{F}_{n}(k \mathbf{r})$ and satisfies eq. (17) (with $k_{n}$ being replaced by the $k$ ). The normali- sation of $\mathbf{E}_{n}(\mathbf{r})$ is then defined as follows:

$$
\begin{aligned}
1= & \int_{R} d \mathbf{r} \varepsilon(\mathbf{r}) \mathbf{E}_{n}^{2}(\mathbf{r}) \\
& -\lim _{k \rightarrow k_{n}} \frac{\int_{R} d S\left(\mathbf{E}_{n} \cdot \frac{\partial \mathbf{E}}{\partial r}-\mathbf{E} \cdot \frac{\partial \mathbf{E}_{n}}{\partial r}\right)}{k_{n}^{2}-k^{2}} .
\end{aligned}
$$

It is again $R$-independent, so that eq. (19) can be evaluated for $R=a$. Furthermore, due to the above mentioned functional behaviour, the limit $k \rightarrow k_{n}$ can be taken explicitly reducing the last term in eq. (19) to

$$
+\frac{1}{2 k_{n}^{2}} \int_{a} d S\left[\mathbf{E}_{n} \cdot \frac{\partial \mathbf{E}_{n}}{\partial r}+r \mathbf{E}_{n} \cdot \frac{\partial^{2} \mathbf{E}_{n}}{\partial r^{2}}-r\left(\frac{\partial \mathbf{E}_{n}}{\partial r}\right)^{2}\right]
$$

To illustrate the 3D case, we take as unperturbed system a dielectric microsphere of radius $a$ and refractive index equal to 1.5. We consider a perturbation (sketched in the left inset of fig. 2) which uniformly increases the refractive index inside the sphere $(\Delta \varepsilon=5$ and 25) leaving its radius unchanged. Since both the unperturbed and perturbed systems have spherical symmetry, the angular momentum $l$ is a good quantum number and can be used to classify the RS. Additionally, eigenmodes can be classified as transverse electric (TE) and transverse magnetic (TM). These TE and TM modes are not mixed by our perturbation. The dielectric microsphere has a rich spectrum containing both leaky and high-Q whispering gallery (WG) modes, the latter having a small imaginary part of $\varkappa_{\nu}$. The difference between these two types of modes becomes more prominent at larger values of $l$, see the spectrum for $l=20$ in fig. 2. For TE modes, the exact solutions (squares and circles) are well reproduced by our method (crosses). To calculate the eigenstates shown in fig. 2, the matrix problem was truncated to $N=200$. The accuracy quickly improves as the matrix dimension $(2 N)$ increases, again with a relative error $\propto N^{-3}$, as is seen in the right inset of fig. 2 .

In conclusion, a Brillouin-Wigner perturbation theory of resonant states in open electromagnetic systems has been developed and validated on exactly solvable $1 \mathrm{D}$ and $3 \mathrm{D}$ examples. Although the wave functions are exponentially growing at large distances and thus cannot be normalised in the usual way, the eigenvalue problem is reduced to the standard linear form, typical for conservative systems. Practically, it only requires diagonalisation of a complex symmetric matrix which consists of the bare spectrum and the perturbation. The simple spectral representation of the Green's function in terms of the calculated perturbed resonant states allows an easy access to measurable quantities such as emission, scattering, and transmission.

The authors thank P. BorRI, A. L. IVANOV and L. Chantada for valuable discussions. E.A.M. acknowledges 
financial support of WIMCS. W.L. acknowledges support from BBSRC, grant BB/E005624.

\section{APPENDIX}

Spectral representation of the Green's function in 1D. - The central quantity for calculating e.g. transmission and emission properties of a dielectric system is the corresponding GF which in 1D satisfies eq. (17) with an arbitrary profile $\varepsilon(z)$ of a dielectric slab embedded in vacuum [i.e. $\varepsilon(z)=1$ for $|z|>a]$. It can be solved in the form

$$
G\left(z, z^{\prime} ; k\right)=\frac{E_{L}\left(z_{<}, k\right) E_{R}\left(z_{>}, k\right)}{W_{L, R}(k)}
$$

with $E_{L}(z, k)$ and $E_{R}(z, k)$ being the left- and rightexponential homogeneous solutions,

$$
\begin{aligned}
& E_{L}(z, k)=e^{-i k z} \text { for } z<-a, \\
& E_{R}(z, k)=e^{+i k z} \text { for } z>+a,
\end{aligned}
$$

so that the GF obeys the BC of outgoing waves at either side, for real positive wave number $k$. The Wronskian $W_{L, R}(k) \equiv E_{L}(z, k) E_{R}^{\prime}(z, k)-E_{L}^{\prime}(z, k) E_{R}(z, k)$ in the denominator takes care of the delta inhomogeneity in eq. (17).

The analytic continuation of the GF into the complex $k$-plane has simple poles at $k_{n}$ where the Wronskian vanishes, $i$. e. where left- and right-exponential solutions agree (up to a factor). This defines the eigenmodes $E_{n}(z) \propto E_{L}\left(z, k_{n}\right) \propto E_{R}\left(z, k_{n}\right)$. They have an exponential behavior on both sides of the slab,

$$
E_{n}(z) \propto \exp \left(i k_{n}|z|\right) \text { for }|z|>a .
$$

For any real dielectric function, the eigenstates come in pairs $\left[E_{n}(z)\right.$ and $\left.E_{n}^{*}(z)\right]$, giving rise to wave numbers which are positioned symmetrically with respect to the imaginary $k$ axis. The states with $\operatorname{Im} k_{n}<0$ which we call RS increase exponentially outside the slab and therefore the orthogonality relation and normalisation have to be modified.

The orthogonality is established by integrating Maxwell's equation (2) for $E_{n}(z)$, multiplied with $E_{m}(z)$, over a finite interval $(-R,+R)$ with $R \geq a$. Integrating by parts the derivative term, and subtracting the interchanged expression $n \leftrightarrow m$ gives

$$
i\left(k_{n}-k_{m}\right)\left[E_{n} E_{m}\right]_{S}+\left(k_{n}^{2}-k_{m}^{2}\right) N_{n m}=0
$$

with the overlap integral

$$
N_{n m}=\int_{-R}^{+R} d z \varepsilon(z) E_{n}(z) E_{m}(z)
$$

and a 'surface' term, $[F]_{S}=F(R)+F(-R)$. Note that the $R$-dependence of the surface term $\left[E_{n} E_{m}\right]_{S}$ and that of the overlap integral $N_{n m}$ cancel each other exactly. We choose normalisation of the $\mathrm{RS}$ in such a way that this property is preserved also for $n=m$ :

$$
N_{n m}-\frac{\left[E_{n} E_{m}\right]_{S}}{i\left(k_{n}+k_{m}\right)}=\delta_{n m},
$$

so that the RS do not depend on $R$. The normalisation constant is chosen such that for bound states (having $\operatorname{Re} k_{n}=0$ and $\operatorname{Im} k_{n}>0$ ) the standard normalisation $N_{n n}=1$ is restored, as here the surface term can be made small for large $R$. A careful investigation shows that the Wronskian derivative with respect to $k$ equals $2 k_{n}$, so that the GF takes the form of eq. (9).

Eigenmodes of the perturbed slab in fig. 1. The profile of the dielectric constant of the perturbed slab sketched in the inset to fig. 1 has the following form

$$
\varepsilon(z)+\Delta \varepsilon(z)=\left\{\begin{array}{cr}
1, & z<-a \\
\varepsilon_{s}, & -a<z<b \\
\varepsilon_{p}, & b<z<a \\
1, & z>a
\end{array}\right.
$$

The eigenmodes are found as solutions of the Maxwell equation (10) with the BC of outgoing waves and have the following explicit form

$$
\mathcal{E}_{\nu}(z)=\left\{\begin{array}{lr}
A e^{-i \varkappa_{\nu} z}, & z<-a, \\
B e^{i \sqrt{\varepsilon_{s}} \varkappa_{\nu} z}+C e^{-i \sqrt{\varepsilon_{s}} \varkappa_{\nu} z}, & -a<z<b, \\
D e^{i \sqrt{\varepsilon_{p}} \varkappa_{\nu} z}+E e^{-i \sqrt{\varepsilon_{p}} \varkappa_{\nu} z}, & b<z<a, \\
F e^{i \varkappa_{\nu} z}, & z>a .
\end{array}\right.
$$

The six coefficients in eq. (A.8) are found (for each $\nu$ ) using the continuity of the $\mathcal{E}_{\nu}(z)$ and $\mathcal{E}_{\nu}^{\prime}(z)$ on the three boundaries $(z=-a, z=b$, and $z=a$ ) and the normalisation condition eq. (A.6). The set of six equations following from the above mentioned BC not only establishes the relations between the coefficients but also leads to the following secular equation for the wave numbers $\varkappa_{\nu}$ :

$$
\alpha \beta f\left(\varkappa_{\nu}\right) g\left(\varkappa_{\nu}\right)-1=\frac{\beta-\alpha}{\alpha \beta-1}\left[\beta g\left(\varkappa_{\nu}\right)-\alpha f\left(\varkappa_{\nu}\right)\right],
$$

where

$$
\alpha=\frac{\sqrt{\varepsilon_{s}}+1}{\sqrt{\varepsilon_{s}}-1}, \quad \beta=\frac{\sqrt{\varepsilon_{p}}+1}{\sqrt{\varepsilon_{p}}-1},
$$

and the functions $f(k)$ and $g(k)$ are defined as follows:

$$
\begin{aligned}
& f(k)=\exp \left[-2 i \sqrt{\varepsilon_{s}} k(a+b)\right], \\
& g(k)=\exp \left[-2 i \sqrt{\varepsilon_{p}} k(a-b)\right] .
\end{aligned}
$$

Derivation for the $3 \mathrm{D}$ case with spherical symmetry. - We consider a dielectric sphere of radius $a$ with a dielectric constant of spherical symmetry, $\varepsilon(r)$, embedded into vacuum. The solutions of Maxwell's equation can be classified into TE and TM modes, and the angular momentum is a conserved quantity. The Green's function can be separated into l-diagonal contributions,

$$
G\left(\mathbf{r}, \mathbf{r}^{\prime} ; k\right)=\frac{1}{r r^{\prime}} \sum_{l=0}^{\infty} G_{l}\left(r, r^{\prime} ; k\right) \frac{2 l+1}{4 \pi} P_{l}(\cos \Theta)
$$

where $\Theta$ denotes the angle between $\mathbf{r}$ and $\mathbf{r}^{\prime}$, and $P_{l}(x)$ are the Legendre polynomials. For simplicity, we consider only the TE case below. The GF obeys the equation

$$
\left[\frac{d^{2}}{d r^{2}}-\frac{l(l+1)}{r^{2}}+k^{2} \varepsilon(r)\right] G_{l}\left(r, r^{\prime} ; k\right)=\delta\left(r-r^{\prime}\right),
$$


which differs from the 1D version eq. (7) only by the centrifugal term $\propto 1 / r^{2}$. For the homogeneous equation, we define a solution $f_{k}(r)$ which is regular at the origin,

$$
f_{k}(r)=r^{l+1} \text { for } r \rightarrow 0
$$

and another one, $g_{k}(r)$, which has outgoing wave BC:

$$
g_{k}(r)=e^{+i k r} \text { for } r \rightarrow \infty .
$$

As in the 1D case, the GF can be constructed using both solutions,

$$
G_{l}\left(r, r^{\prime} ; k\right)=\frac{f_{k}\left(r_{<}\right) g_{k}\left(r_{>}\right)}{W\left\{f_{k}, g_{k}\right\}} .
$$

The analytic continuation into the complex $k$ plane has poles wherever the Wronski determinant has zeroes which defines the wave numbers of the resonant modes,

$$
W\left\{f_{k}, g_{k}\right\}=0 \text { at } k=k_{n} .
$$

At $k_{n}$, both solutions $f_{k}$ and $g_{k}$ agree up to a factor. Integrating $G_{l}\left(r, r^{\prime} ; k^{\prime}\right) /\left(k^{\prime}-k\right)$ over a large circle in the complex $k^{\prime}$ plane gives zero, but can be decomposed into pole contributions with the result

$$
G_{l}\left(r, r^{\prime} ; k\right)=\sum_{n} \frac{f_{k_{n}}(r) g_{k_{n}}\left(r^{\prime}\right)}{\left(k-k_{n}\right) w_{n}}
$$

(note that in $3 \mathrm{D}$, there is no trivial pole at $k=0$ ). The Wronskian derivative, $w_{n}=d W /\left.d k\right|_{k=k_{n}}$, is calculated integrating Maxwell's equation by parts over the finite interval $(0, R)$ with $R \geq a$ which gives

$$
\begin{aligned}
\frac{d W\left\{f_{k}, g_{k}\right\}}{d k}= & f_{k}(R) \frac{d g_{k}^{\prime}(R)}{d k}-f_{k}^{\prime}(R) \frac{d g_{k}(R)}{d k} \\
& +2 k \int_{0}^{R} d r \varepsilon(r) g_{k}(r) f_{k}(r),
\end{aligned}
$$

owing to the $k$-independent regular solution eq. (A.15) at $r \rightarrow 0$. A further simplification is possible due to $R \geq a$ where $\varepsilon(r) \equiv 1$ holds. Here, the outgoing wave solution depends on $r$ and $k$ only in product form, $g_{k}(r)=\tilde{g}(x=$ $k r$ ). Then, the first term on the r.h.s. of eq. (A.20) can be rewritten as

$$
f_{k}(R)\left[\tilde{g}^{\prime}(x)+x \tilde{g}^{\prime \prime}(x)\right]-\left.f_{k}^{\prime}(R) R \tilde{g}^{\prime}(x)\right|_{x=k R} .
$$

At the poles, we have $f_{k_{n}}(r)=c_{n} \cdot g_{k_{n}}(r)$, and can take $g_{n}(r) \equiv g_{k_{n}}(r)$ everywhere,

$$
\begin{aligned}
w_{n}= & \tilde{g}(x) \tilde{g}^{\prime}(x)+\left.x\left[\tilde{g}(x) \tilde{g}^{\prime \prime}(x)-\left(\tilde{g}^{\prime}(x)\right)^{2}\right]\right|_{x=k_{n} R} \\
& +2 k_{n} \int_{0}^{R} d r \varepsilon(r) g_{n}^{2}(r)
\end{aligned}
$$

in conjunction with

$$
G_{l}\left(r, r^{\prime} ; k\right)=\sum_{n} \frac{g_{n}(r) g_{n}\left(r^{\prime}\right)}{\left(k-k_{n}\right) w_{n}} .
$$

Equation (A.22) can be considered as the proper normalisation of the resonant modes. Its l.h.s. is formally equivalent to their orthogonality (valid for $k_{n} \neq k_{m}$ ),

$$
\begin{aligned}
0= & \frac{g_{n}(R) g_{m}^{\prime}(R)-g_{n}^{\prime}(R) g_{m}(R)}{k_{m}-k_{n}} \\
& +\left(k_{n}+k_{m}\right) \int_{0}^{R} d r \varepsilon(r) g_{n}(r) g_{m}(r),
\end{aligned}
$$

if the limit $k_{m} \rightarrow k_{n}$ is performed.

\section{REFERENCES}

[1] Schrödinger E., Ann. Physik, 80 (1926) 437.

[2] Brillouin L., J. Phys. Radium, 3 (1932) 373.

[3] Gamow G., Z. Phys., 51 (1928) 204; Z. Phys., 52 (1929) 510.

[4] Zel'dovich YA., Zh. Eksp. Teor. Fiz., 39 (1960) 776. [Sov. Phys.-JETP, 12 (1961) 542].

[5] BaZ' A., Zel'dovich Ya. and Perelomov A., Scattering, Reactions and Decay in Nonrelativistic Quantum Mechanics (U. S. Department of Commerce, Washington, D. C.) 1969.

[6] Weinstein L. A., Open Resonators and Open Waveguides (Golem Press, Boulder, Colorado) 1969.

[7] More R. M., Phys. Rev. A, 4 (1971) 1782.

[8] More R. M. and Geruuoy E., Phys. Rev. A, 7 (1973) 1288.

[9] Olshansky R., Rev. Mod. Phys., 51 (1979) 341.

[10] Tikhodeev S. G., Yablonskit A. L., Muljarov E. A., Gippius N. A. and Ishinara T., Phys. Rev. B, 66 (2002) 045102.

[11] Leung P. T., Liu S. Y., Tong S.S. and Young K., Phys. Rev. A, 49 (1994) 3068.

[12] Leung P. T. and PAng K. M., J. Opt. Soc. Am. B, 13 (1996) 805.

[13] Lai H. M., Leung P. T., Young K., Barber P. W. and Hill S. C., Phys. Rev. A, 41 (1990) 5187.

[14] Dettmann C. P., Morozov G. V., Sieber M. and H. Waalkens , Phys. Rev. A, 80 (2009) 063813.

[15] Bang J., Gareev F. A., Gizzatkulov M. H. and S. A. Goncharov, Nuclear Physics A, 309 (1978) 381.

[16] Lind P., Phys. Rev. C, 47 (1993) 1903.

[17] M. A. M. De Aguiar, Phys. Rev. A, 48 (1993) 2567.

[18] Newton R., J. Math. Phys., 1 (1960) 319.

[19] C. V. Sukumar, Am. J. Phys., 58 (1990) 561.

[20] Bang J. and Gareev F. A., Lett. Nuovo Cimento, 32 (1981) 420.

[21] A quantum-mechanical analogue of eq. (15) can be found in refs. 15] and [16.

[22] Watson D. K., Phys. Rev. A, 34 (1986) 1016.

[23] This can be shown explicitly expanding the $\mathbf{E}_{n}(\mathbf{r})$ into the complete set of vector spherical harmonics 24].

[24] Stratton J.A., Electromagnetic theory (McGraw-hill Book Company, New York and London) 1941. 